\title{
Chalcogenide glass hollow-core microstructured optical fibers
}

\author{
Vladimir S. Shiryaev ${ }^{1,2 *}$ \\ G.G. Devyatykh Institute of Chemistry of High-Purity Substances of the Russian Academy of Sciences, Nizhny Novgorod, Russia \\ ${ }^{2}$ N.I. Lobachevski Nizhny Novgorod State University, Nizhny Novgorod, Russia
}

\section{Edited by:}

Petra Granitzer, University of Graz,

Austria

Reviewed by:

Tatiana S. Perova, Trinity College

Dublin, Ireland

Roman Golovchak, Austin Peay State

University, USA

*Correspondence:

Vladimir S. Shiryaev, G.G. Devyatykh

Institute of Chemistry of High-Purity

Substances of the Russian Academy

of Sciences, 49 Tropinin Street,

Nizhny Novgorod 603950, Russia

e-mail:shiryaev@ihps.nnov.ru
The recent developments on chalcogenide glass hollow-core microstructured optical fibers (HC-MOFs) are presented. The comparative analysis of simulated optical properties for chalcogenide HC-MOFs of negative curvature with different size and number of capillaries is given. The technique for the manufacture of microstructured chalcogenide preforms, which includes the assembly of the substrate glass tube and 8-10 capillaries, is described. Further trends to improve the optical transmission in chalcogenide negative curvature hollow-core photonic crystal fibers are considered.

Keywords: chalcogenide glass, microstructured fibers, optical loss, mid-IR, preform

\section{INTRODUCTION}

The hollow-core microstructured optical fibers (HC-MOFs) (Yablonovitch, 1987; Knight et al., 1996) have attracted much attention in optical applications over the past decade as they exhibit many unique optical properties such as controlled dispersion, endlessly single-mode operation, supercontinuum generation, and soliton propagation (Birks et al., 1997; Monro et al., 1999; Revathi et al., 2014; Skryabin and Wadsworth, 2010; Wadsworth et al., 2001) over a wide range of wavelengths that cannot be realized in conventional step-index fibers. The light guidance in HC-MOF fiber is based on the mechanism of photonic band gap (PBG) guidance. The PBG mechanism has the capabilities to control the guidance of light with a certain frequency band (Knight et al., 1998; Cregan et al., 1999). In HC-MOFs, core localized modes can exist with mode effective indices $b / k_{0}$ (where $b$ is the propagation constant and $k_{0}$ is the wavenumber in free space) that are lower than the mean index of the microstructured cladding (Cregan et al., 1999), so it is possible to guide the light in air.

The theoretical losses in HC-MOFs can be very low, because the material absorption and Rayleigh scattering in air are negligible compared to glass (Knight et al., 1996). Special potential is accorded to HC-MOFs with negative curvature of refractive index. Experiments on silica fibers of that type have shown not only the possibility of achieving low optical losses, but also a considerable extension of the transmission region to longer wavelengths in comparison with conventional step-index optical fibers (Knight et al., 1996; Pryamikov et al., 2011).

There are many reasons for interest in guiding light in a hollowcore. For example, the effective single-mode guidance of light was demonstrated in HC-MOF with six-layer cladding structure made from a lead-silicate "soft" glass (Jiang et al., 2011). Also, in gasfilled HC-MOFs, there is a strong interaction between the light and the gas which fills the core, giving a lower energy threshold for
Raman amplification (Benabid et al., 2005). HC-MOFs are also of interest for fiber sensors and applications requiring single-mode guidance in a large mode area, such as power delivery, fluid and gas-filled devices, and particle transport (Benabid, 2006).

In recent years, great attention has been paid to development of the mid-infrared (IR) region, which is due to numerous potential applications in analytical IR spectroscopy, pyrometers, and transmission of IR lasers. Among the wide variety of IR glasses, for mid-IR MOFs, non-silica compound glasses like chalcogenide glasses have the best prospects. Chalcogenide glasses are based on sulfur, selenium, tellurium, and the addition of other elements such as arsenic, germanium, antimony, gallium, etc. They are characterized by a number of significant advantages, such as a wide transmittance range $(1-12 \mu \mathrm{m})$, low intrinsic losses in the midIR, low phonon energy, and the absence of free-carrier effects (Snopatin et al., 2009b). The large refractive index of chalcogenide glasses (compared to other glasses) of 2.4-3.0 opens up the possibility of achieving compact non-linear devices (Hu et al., 2014; Zhang et al., 2014; He et al., 2012). The non-linear refractive index $n_{2}$ in chalcogenide glasses is by $(100-1000)$ times higher than in silica glass. These two properties are more suitable for fiber-based photonic wire devices (He et al., 2012).

There are a large number of technical problems in fabrication of HC-MOFs based on chalcogenide glasses. Several research groups from different countries are involved in tackling these problems. The main efforts are focused on the development of optimum design and geometric parameters of MOF structure, the increase in the chemical and phase purity of bases of chalcogenide glass, the improvement of drawing technique. The results of these studies are not widely presented in the literature, since the development of solutions to the abovementioned problems is at a very early stage. This review presents the modern status of developments on chalcogenide glass HC-MOFs. 


\section{THE FIRST EXPERIMENTS ON PREPARING THE CHALCOGENIDE HC-MOFs}

The development of near- and mid-IR hollow-core chalcogenide MOFs is an important and urgent task because of the theoretical possibility to achieve optical losses, which are lower than material losses, an expansion of transmission range, as well as to transmit high-power $\mathrm{CO}$ and $\mathrm{CO}_{2}$-laser radiation (Pryamikov et al., 2011; Temelkuran et al., 2002).

Therefore, after the first publication on MOF of Ga-La-S system in 1998 (Monro et al., 2000), an interest in the world in the preparation and study of such fibers of different chalcogenide glasses, as a solid-core and hollow-core MOFs, has increased significantly. Published data on chalcogenide MOFs with a hollow-core is limited because the production of such fibers is a rather difficult technical challenge.

In published papers on preparing chalcogenide MOFs with a solid-core, the following techniques of preform manufacturing have been used: "stack and draw" (assembly of capillaries inside the substrate tube) (Brilland et al., 2006; Desevedavy et al., 2008), molding (with use of silica glass pattern of the specified photonic crystal design) (Coulombier et al., 2010; Conseil et al., 2011), and drilling (El-Amraoui et al., 2010). Usually, the thin capillaries are drawn from the chalcogenide glass tube prepared by centrifugal casting. However, the additional heat treatment can provoke crystallization of the glass and increase the excess optical loss in the resulting fibers. The main disadvantage of the drilling method to make holes in the substrate chalcogenide glass tube is the formation of broken surface layers of the holes.

There are one-dimensional (1-D) (hybrid Bragg photonic crystal fiber) and two-dimensional (2-D) hollow fibers made of chalcogenide glasses.

One-dimensional MOF is a hybrid structure consisting of dielectric mirrors to guide light inside the fibers. A dielectric mirror is basically simultaneous layers of quarter wave stacks, made of thermo mechanically compatible materials with high-refractiveindex difference. The modification of thin dielectric mirror layers designed at the inner surface of a hollow fiber allows this fiber to guide a certain spectrum of light through air efficiently.
The first HC-MOFs were hybrid Bragg fibers (Yeh et al., 1978) composed of successive circular layers presenting two different refractive indices. An example of such hollow-core Bragg fiber composed of layers of $\mathrm{As}_{2} \mathrm{Se}_{3}$ chalcogenide glass with a refractive index of 2.8 and high glass-transition temperature thermoplastic polymer poly(ether sulfone) (PES) having a refractive index of 1.55 was described in paper (Temelkuran et al., 2002) (Figure 1). A hollow preform from these two materials was made by vapor depositing an $\mathrm{As}_{2} \mathrm{Se}_{3}$ coating on a thin sheet of PES, with the subsequent rolling the $\mathrm{As}_{2} \mathrm{Se}_{3} / \mathrm{PES}$ sheet into a fiber preform. Confinement of light in the hollow-core is provided by the large photonic bandgaps established by the multiple alternating submicrometer-thick layers of a high-refractive-index glass and a low-refractive-index polymer. The fundamental and high-order transmission windows are determined by the layer dimensions and can be change in wavelength range from 0.75 to $10.6 \mu \mathrm{m}$. The transmission losses are found to be less than $1.0 \mathrm{~dB} / \mathrm{m}$. The large photonic bandgaps result in very short electromagnetic penetration depths within the layer structure, significantly reducing radiation and absorption losses. The maximum laser power density coupled into these fibers was approximately $300 \mathrm{~W} / \mathrm{cm}^{2}$.

Another method to prepare a 1-D photonic bandgap structure was proposed in paper (Gibson and Harrington, 2004). The authors extruded a stack of alternating plates of $\mathrm{As}_{2} \mathrm{Se}_{3} / \mathrm{PSU}$ (polysulfone) through a die into a hollow-core preform with dielectric multi-layers.

The most common design of 2-D MOF introduced by Birks et al. (1997) can be described as a hollow-core fiber in which the cross-section is a periodic array of air holes placed along the length of the fiber. The origin of the band gap for 2-D MOFs is similar to the 2-D band gaps investigated for planar light-wave circuits. The most common geometry for 2-D MOFs is presented in Figure 2A.

The first all-chalcogenide hollow-core photonic crystal fibers (HC-MOF) was reported in paper (Desevedavy et al., 2010). These fibers composed of six rings of holes and regular microstructures with Kagome and hexagonal lattices were fabricated by the "stack and draw" technique from $\mathrm{Te}_{20} \mathrm{As}_{30} \mathrm{Se}_{50}$ glass (Figure 2B). Unfortunately, the targeted HC-MOF profile was not reached and

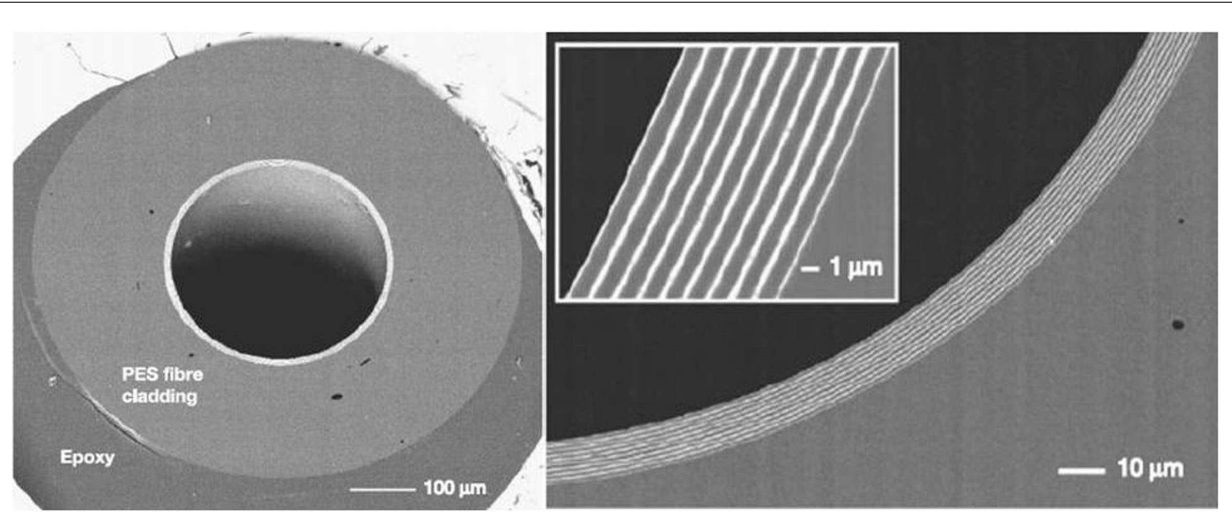

FIGURE 1 | Cross-sectional SEM micrographs at various magnifications of hollow cylindrical multi-layer fiber mounted in epoxy. The hollow-core appears black, the PES layers and cladding gray, and the $\mathrm{As}_{2} \mathrm{Se}_{3}$ layers bright white (Temelkuran et al., 2002). 


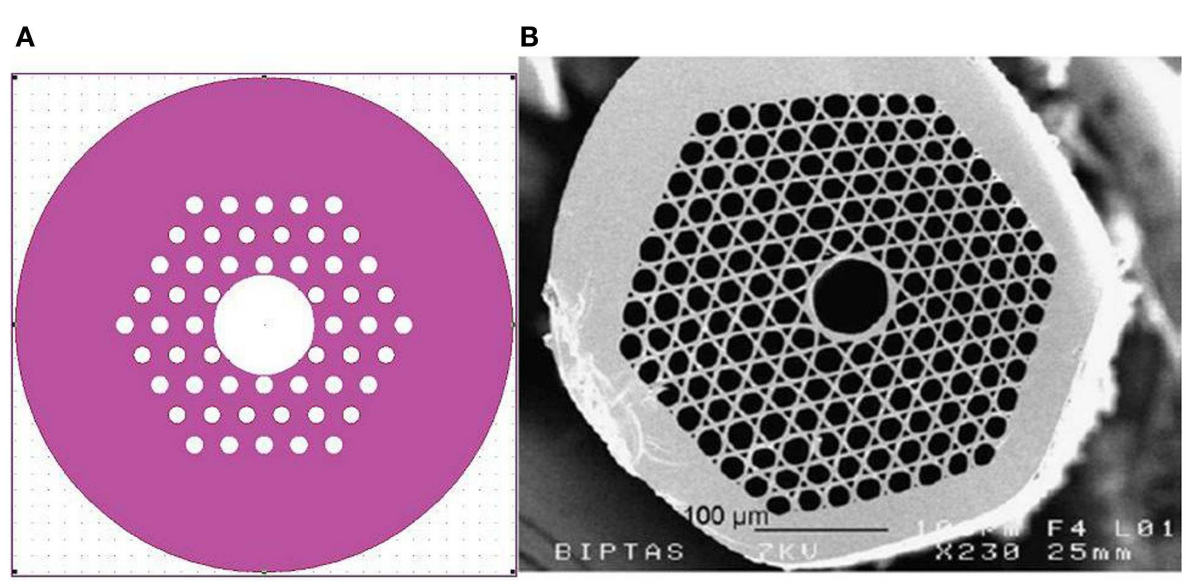

FIGURE 2 | (A) Geometry for 2-D PCFs; (B) Te ${ }_{20} \mathrm{As}_{30} \mathrm{Se}_{50}$ hollow-core PCF profiles with Kagome lattice cladding (Desevedavy et al., 2010).

no propagation was observed in the core fibers between 2 and $20 \mu \mathrm{m}$.

By now, the optical loss in step-index chalcogenide glass fibers has been reduced to $1.5-2 \mathrm{~dB} / \mathrm{m}$ at $\lambda=10.6 \mu \mathrm{m}$ due to a decrease of the oxide impurity content causing absorption in 7.5-15 spectral region (Nishii et al., 1992; Katsuyama and Matsumura, 1986; Shiryaev et al., 2005). The maximum power transmitted through a $1-\mathrm{m}$ length of such a fiber was $10.7 \mathrm{~W}$, when an anti-reflection coating and water cooling were used (Nishii et al., 1992). Better transmission results have been achieved with hollow-core fibers, in which radiation power is transmitted through the air. A hollowcore polymer fiber with a cladding in the form of a multi-layer Bragg mirror demonstrated a loss below $1 \mathrm{~dB} / \mathrm{m}$ at $\lambda=10.6 \mu \mathrm{m}$ (Temelkuran et al., 2002).

\section{CHALCOGENIDE NEGATIVE CURVATURE HOLLOW-CORE PHOTONIC CRYSTAL FIBERS. THEORY}

To develop a model chalcogenide HC-MOF (Kosolapov et al., 2011), a relatively simple design of photonic crystal structure of eight capillaries within the substrate tube was used (Figure 3), as tested previously for silica glass (Pryamikov et al., 2011). The ratio of the inner and outer diameters of the capillaries should be from 0.8 to 0.9 . These negative curvature hollow-core photonic crystal fibers (NCHCFs) with a negative curvature of the core boundary belongs to the type of HC-MOFs that do not support PBGs. Such NCHCFs have relatively high transmission losses in comparison with PBG HC-MOFs, but possess a larger bandwidth, because the air-core localized modes are coupled only weakly with the cladding modes in the low loss wavelength regions. The high loss wavelengths in the transmission spectrum correspond to the avoided crossing of the air-core modes and the cladding modes.

The simulation of the optimal design of the NCHCF was carried out by the FemLab 3.1 software, by means of the finite element method. Figure 4 gives the theoretical losses of the fundamental $\mathrm{HE}_{11}$ air-core mode for $\mathrm{As}_{2} \mathrm{~S}_{3}$ and $\mathrm{As}_{30} \mathrm{Se}_{50} \mathrm{Te}_{20}$ glass fibers for the structure illustrated in Figure 3, with air-core diameter $D_{\text {core }}$ of $223 \mu \mathrm{m}$, the outer diameter of capillaries of $178 \mu \mathrm{m}$, the

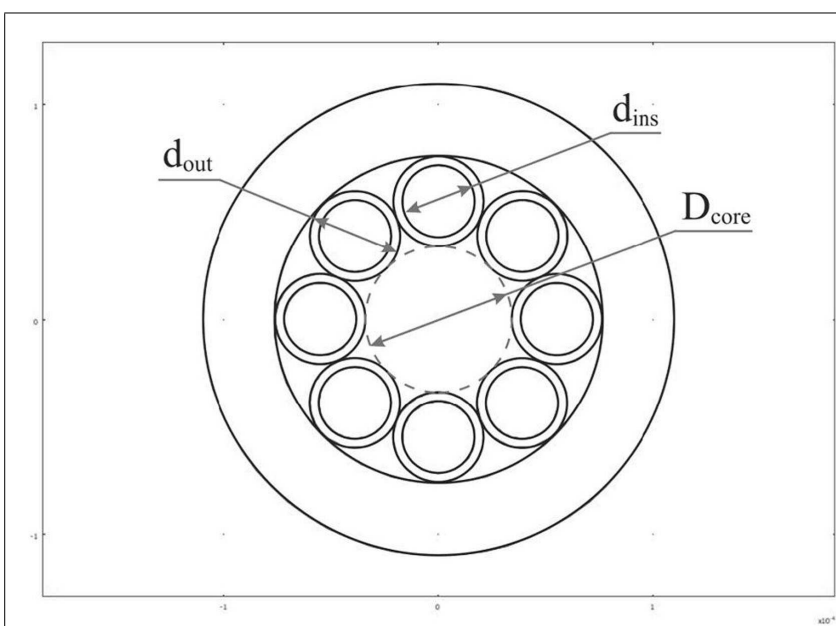

FIGURE 3 | HC-MOF with negative curvature of the core with cladding consisting of one row of capillaries (Pryamikov et al., 2011).

ratio of the inner and outer diameters of the capillaries of 0.8 (Shiryaev et al., 2014a). It can be seen that the spectrum of ideal fiber with negative curvature of the hollow-core is irregular, the optical losses change in the transmission windows from a minimum value of $0.001 \mathrm{~dB} / \mathrm{m}$ to a maximum value of $100 \mathrm{~dB} / \mathrm{m}$. Such irregular behavior of optical losses can be explained by the weak coupling of the core modes with the dielectric modes having a high Azimuthal dependence. Theoretical spectra of optical losses in ideal NCHCFs based on $\mathrm{As}_{2} \mathrm{~S}_{3}$ and $\mathrm{As}_{30} \mathrm{Se}_{50} \mathrm{Te}_{20}$ glasses are similar, but transmittance bands for $\mathrm{As}_{2} \mathrm{~S}_{3}$ are wider.

The calculated bending losses for fiber of arsenic sulfide were calculated to be about 3-4 times lower as compared with $\mathrm{As}_{30} \mathrm{Se}_{50} \mathrm{Te}_{20}$ (Figure 5) (Shiryaev et al., 2014a). The losses of the fundamental $\mathrm{HE}_{11}$ air-core mode for small bending radii are characterized by the resonance behavior. The theoretical losses in $\mathrm{As}_{2} \mathrm{~S}_{3}$ $\mathrm{NCHCF}$ in the field of $\mathrm{CO}_{2}$-laser radiation at a wavelength of $10.6 \mu \mathrm{m}$ were about $0.1 \mathrm{~dB} / \mathrm{m}$. 


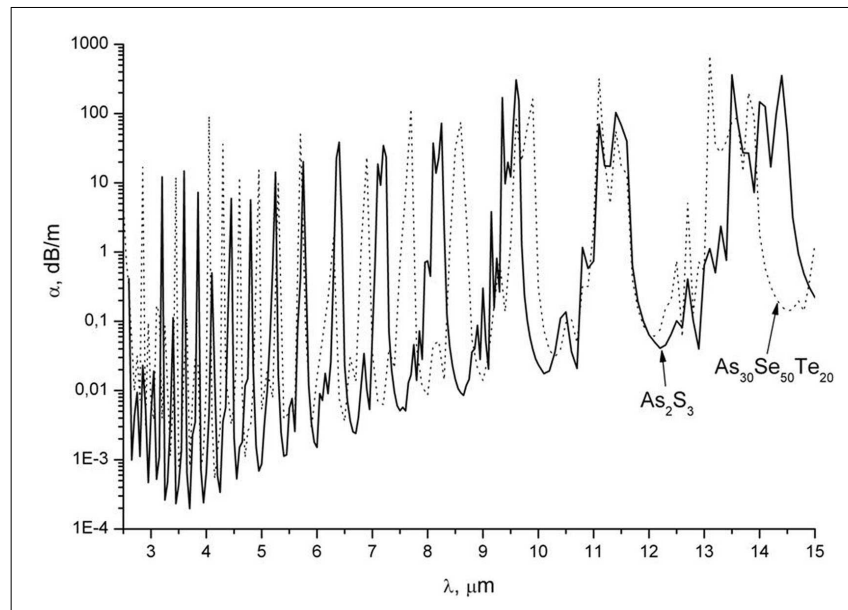

FIGURE 4 |The calculated transmission bands for ideal NCHCF (eight capillaries; $D_{\text {core }}=223 \mu \mathrm{m}, d_{\text {in }} / d_{\text {out }}=0.8$ ) for $\mathrm{As}_{2} \mathrm{~S}_{3}$ (solid line) and $\mathrm{As}_{30} \mathrm{Se}_{50} \mathrm{Te}_{20}$ (dotted line) glasses (Shiryaev et al., 2014b).

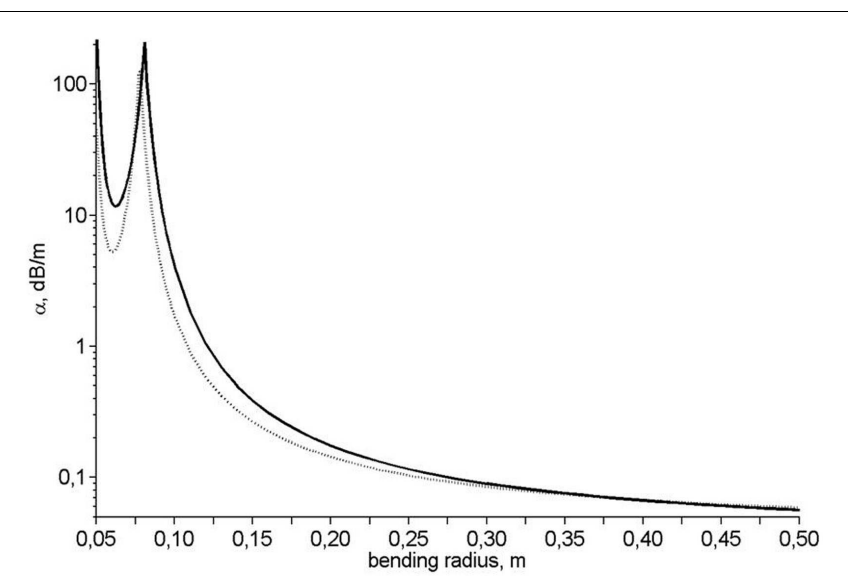

FIGURE 5 | Calculated dependence of the bending losses of the fundamental $\mathrm{HE}_{11}$ air-core mode on bending radius at $\lambda=10.3 \mu \mathrm{m}$ for $\mathrm{As}_{2} \mathrm{~S}_{3}$ (dotted line) and $\mathrm{As}_{30} \mathrm{Se}_{50} \mathrm{Te}_{20}$ (solid line) glasses (Shiryaev et al., 2014b).

In paper (Pryamikov et al., 2012), four types of HC-MOFs with the claddings consisting of $6,8,10$, and 12 capillaries were considered. The calculations were carried out for two values of the glass refractive indices $n=2.4,2.8$ and with three values of $d_{\text {in }} / d_{\text {out }}=0.8,0.85,0.9$. All calculations were made in the narrow spectral region near $\lambda=10.6 \mu \mathrm{m}$. It has been shown that the achievement of a low loss waveguide regime for HC MFs with the cladding consisting of capillaries is complicated multi parameter task. All the parameters characterizing the HC MFs such as $D_{\text {core }}$ (hollow-core diameter), $d_{\text {in }}$ (inner diameter of capillaries), $d_{\text {out }}$ (outside diameter of capillaries), $n, N$ (number of the capillaries in the cladding) have an effect on the waveguide regime in the considered spectral range. In this way, two main factors affect the loss level of the HC-MOFs: the density of eigen states of the individual capillary and the discrete rotational symmetry of the core boundary. The first factor is determined by geometry parameters of a capillary and the value of a glass refractive index. The second factor is connected to the symmetry of the capillary arrangement in the cladding. It was established that a balance between the number of capillaries and the air-core diameter should be found.

The authors of paper (Wei et al., 2014) have carried out the simulating analysis for chalcogenide negative curvature hollowcore MOFs with different capillary wall thickness $(t)$ to diameter ratios, $t / d_{\text {out }}$ and different number of capillaries, $N$, of $8,10,12$, and 14. They have found that leakage loss and power ratio in the glass decrease as the number of capillaries increases or tube wall thickness to diameter ratio decreases.

\section{PREPARATION OF PREFORMS FOR CHALCOGENIDE NEGATIVE CURVATURE FIBERS}

The description of preparation of fiber preforms for chalcogenide negative curvature fibers is given in papers (Kosolapov et al., 2011) and (Shiryaev et al., 2014a). These performs were manufactured by the "stack and draw" technique from a substrate tube and 8 or 10 capillaries of the same chalcogenide glass. For this, high-purity chalcogenide glasses with low tendency to crystallization such as $\mathrm{As}_{2} \mathrm{~S}_{3}, \mathrm{As}_{40} \mathrm{~S}_{30} \mathrm{Se}_{30}$, and $\mathrm{As}_{30} \mathrm{Se}_{50} \mathrm{Te}_{20}$ were used.

The $\mathrm{As}_{2} \mathrm{~S}_{3}$ glass was produced by melting purified arsenic monosulfide $\left(\mathrm{As}_{4} \mathrm{~S}_{4}\right)$ as the arsenic-containing component with the required amount of elementary sulfur in a sealed silica ampoule (Snopatin et al., 2009a). The preparation method of high-purity $\mathrm{As}_{40} \mathrm{~S}_{30} \mathrm{Se}_{30}$ and $\mathrm{As}_{30} \mathrm{Se}_{50} \mathrm{Te}_{20}$ glasses included the purification of glass-forming chalcogenides by chemical distillation methods, the melting of the purified fractions up to homogeneous state, and the melt solidification (Shiryaev et al., 2004; Shiryaev and Balda, 2006).

The obtained $\mathrm{As}_{40} \mathrm{~S}_{30} \mathrm{Se}_{30}$ and $\mathrm{As}_{30} \mathrm{Se}_{50} \mathrm{Te}_{20}$ glasses had a low content of the limiting impurities: hydrogen $-<0.06 \mathrm{ppm} w \mathrm{w}$, oxygen $-0.2 \mathrm{ppm} w \mathrm{t}$, carbon $-1 \mathrm{ppm} \mathrm{wt}$, silicon $-0.5 \mathrm{ppm} \mathrm{wt}$ (as follows from laser mass spectroscopy and IR spectroscopy). The content of limiting impurities in $\mathrm{As}_{2} \mathrm{~S}_{3}$ glass was as follows: carbon $-<0.1 \mathrm{ppm} w \mathrm{w}$, hydrogen as $\mathrm{SH}$ group $-<0.5 \mathrm{ppm} w \mathrm{wt}$ silicon $-\leq 0.5 \mathrm{ppm} w \mathrm{wt}$, metals $-<0.1 \mathrm{ppm}$ wt. The optical loss spectra of mono-index fibers drawn from these glasses are given in the Figures 6A,B.

The substrate tubes with the outer diameter of $16 \mathrm{~mm}$, the inner diameter of $11 \mathrm{~mm}$, and the length of 140-170 mm (Figure 7A) were fabricated by centrifugal casting of the chalcogenide glass melt inside an evacuated silica tube (Kosolapov et al., 2011; Shiryaev and Churbanov, 2013; Shiryaev et al., 2014a).

The capillaries with the outer diameter of 2.4-3.2 mm were prepared by the double crucible method from the chalcogenide glass melt. For this, the chalcogenide glass was placed in the cladding crucible, while the core crucible remained empty. The capillary wall thickness was determined by the geometrical dimensions of the double crucible die, the excess inert gas pressure over the melt, and the pressure inside the capillary. The cross-sections of the capillaries had a coaxial geometry with a ratio of internal and outside diameters of $0.8-0.9$. Concentricity of obtained capillaries was over $80 \%$.

The final preform consisted of the substrate tube and 8 or 10 capillaries forming a complete azimuthally symmetric layer of holes on the inner surface of the tube (Figures $7 \mathbf{B}$ and $\mathbf{8 A}$ ). 

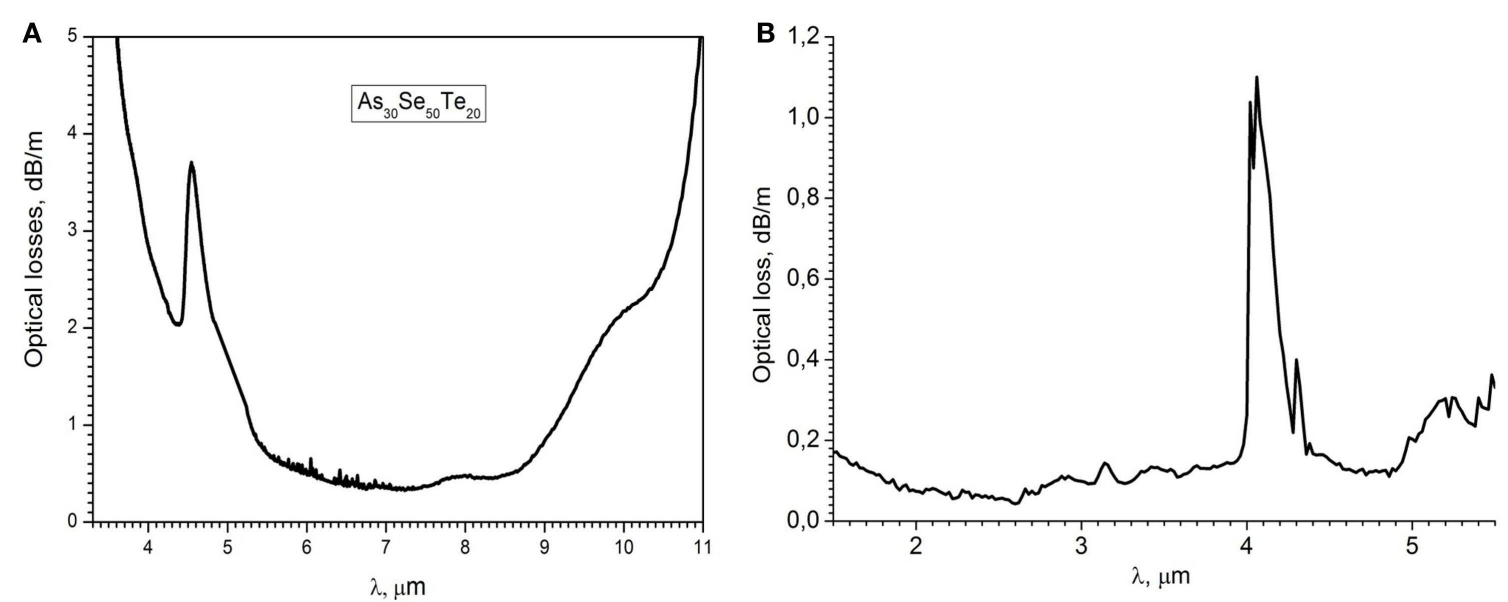

FIGURE 6 | Optical losses of mono-index $A s_{30} \mathrm{Se}_{50} \mathrm{Te}_{20}$ (A) and $A s_{2} S_{3}$ (B) glass fibers (Shiryaev et al., $2014 \mathrm{~b}$ ).

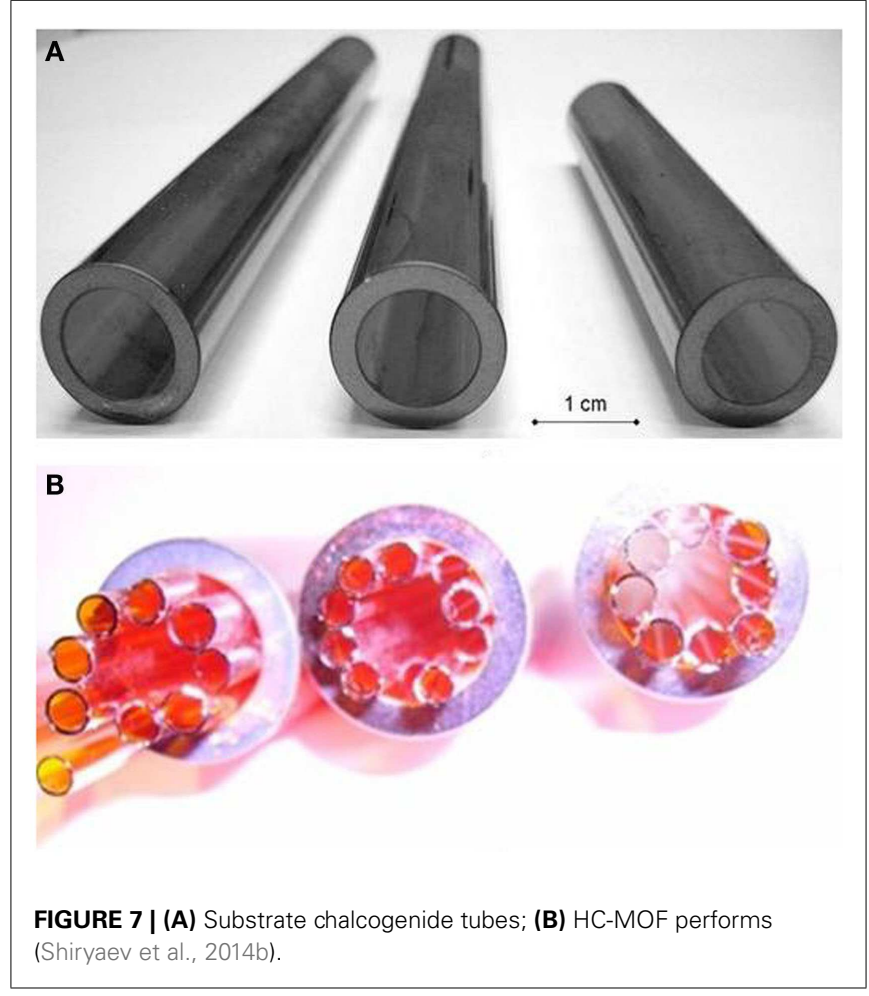

ADHESION OF CHALCOGENIDE GLASSES TO SILICA GLASS

The realization of the centrifugal casting method of chalcogenide melt inside a silica ampoule is associated with the need to determine the time-temperature conditions to obtain the perfect substrate tube. However, the substrate chalcogenide tubes, especially based on arsenic sulfide glass, were found to crack due to the strong adhesion of chalcogenide glass to silica glass at annealing temperatures. Therefore, to determine the optimal conditions for manufacturing the perfect substrate chalcogenide tubes, the investigation of temperature dependence of chalcogenide glass adhesion to silica glass was carried out (Shiryaev et al., 2014b; Shiryaev
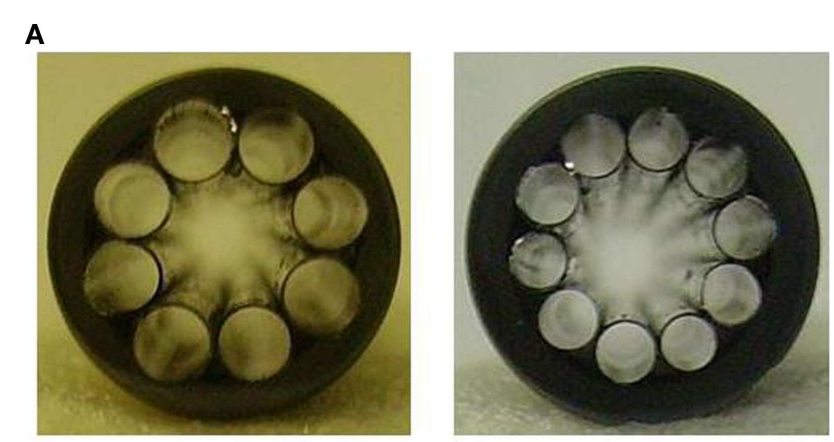

B
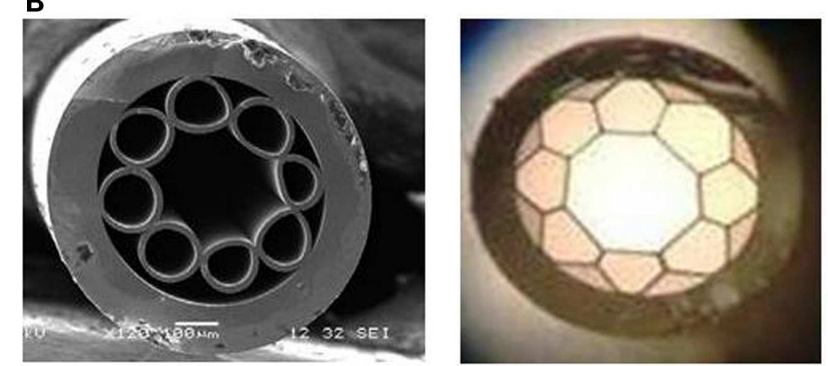

FIGURE 8 | Cross-sections of preforms (A) and microstructured fibers

(B) (Shiryaev and Churbanov, 2013).

et al., 2015). It was carried out by the steady detachment method. This method is used to measure the force required to separate the adhesive from the substrate simultaneously in all areas of contact.

Results of the temperature dependence of separation tension of six chalcogenide glasses from silica glass are shown in Figure 9 (Shiryaev et al., 2015). These dependences show that the adhesion strength has the maximum near $T_{\mathrm{g}} \pm 10^{\circ} \mathrm{C}$ of chalcogenide glasses. Adhesion strength exceeds the value of $2500 \mathrm{kPa}$ for $\mathrm{As}_{40} \mathrm{~S}_{60}$ and $\mathrm{As}_{40} \mathrm{~S}_{30} \mathrm{Se}_{30}$ glasses at temperatures above $(170-185)^{\circ} \mathrm{C}$.

The adhesive strength was determined to increase with contact temperature, as well as with the exposure time of assembly 
up to the stationary value, depending on the contact temperature. The investigation of chalcogenide glass composition on the value of adhesion to the silica glass showed, that the adhesion strength of chalcogenide glasses to silica glass increases with decrease in chalcogen molar mass and with increase in chalcogen content

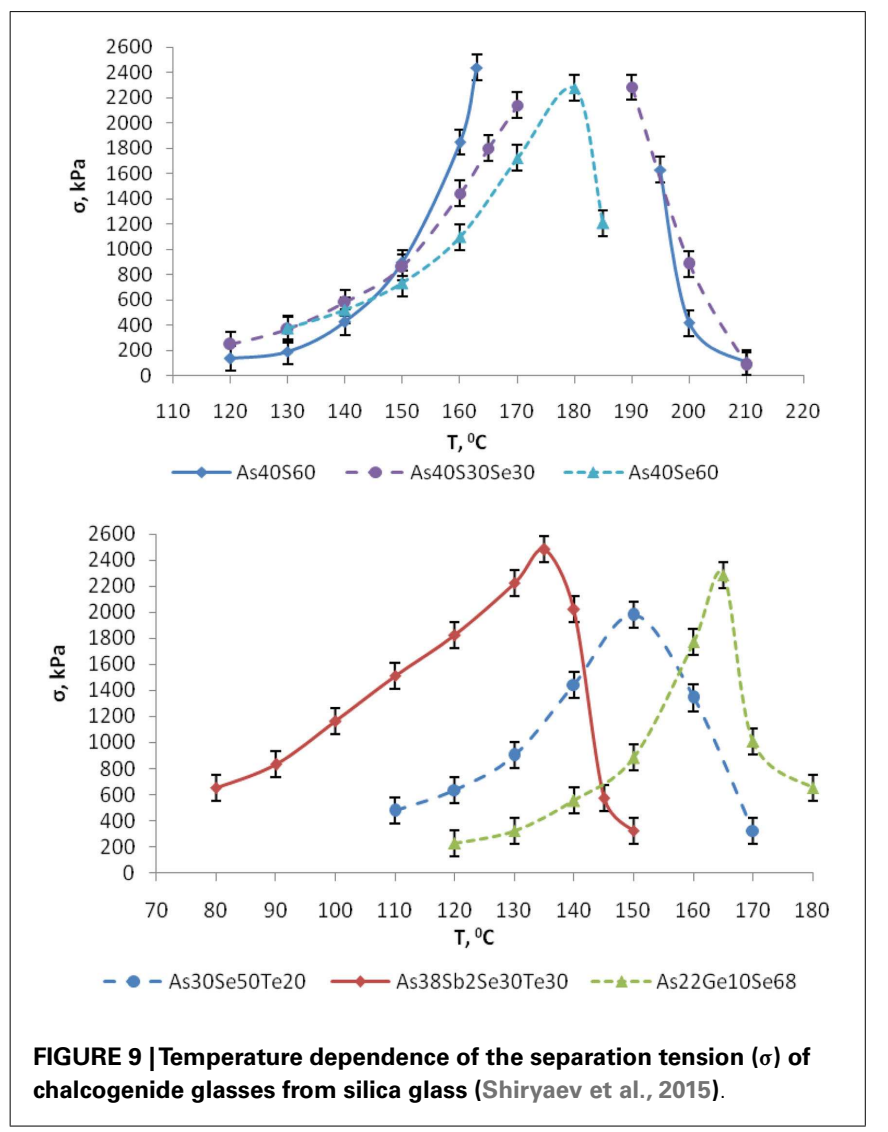

in the glass composition. The obtained results made it possible to explain the observed experimental data for forming chalcogenide glass tube preforms and to optimize the process for their preparation.

Adhesion is the critical parameter during the annealing of the samples in the form of rods and tubes near the glass-transition temperature $\left(185^{\circ} \mathrm{C}\right)$. To prevent the destruction of preforms during the cooling process, a separation of the surface of the chalcogenide glass sample from the walls of the silica ampoule, executing a forced local cooling of interface of chalcogenide and silica glasses, must be used.

\section{PREPARATION OF CHALCOGENIDE NEGATIVE CURVATURE FIBERS}

The prepared preforms were used to draw NCHCFs (Kosolapov et al., 2011; Shiryaev and Churbanov, 2013; Shiryaev et al., 2014a). For this, the overpressure of dry inert gas (argon) was established in each capillary and inside of the substrate tube. The preforms with an outside diameter of $16 \mathrm{~mm}$ were drawn in fibers with diameter of 700-900 $\mu \mathrm{m}$. To prepare a defined structure of NCHCF, the conditions of fiber drawing were determined by experiment. A high temperature of fiber drawing resulted in the random distribution of capillaries inside the substrate tube (Figures 10A-C). In case of $\mathrm{As}_{2} \mathrm{~S}_{3}$ glass, the NCHCF had a structure close to the defined one, at a drawing temperature of $310^{\circ} \mathrm{C}$ and an excess argon pressure in capillaries of $4000 \mathrm{~Pa}$. The capillary rings had no discontinuities, and the capillaries themselves had deviations from the original spherical shape due to conditions of fiber drawing (Figures 8B and 10D,E).

\section{OPTICAL TRANSMISSION OF CHALCOGENIDE NEGATIVE CURVATURE FIBERS}

The fiber loss spectra of as-drawn NCHCFs (Kosolapov et al., 2011; Shiryaev and Churbanov, 2013; Shiryaev et al., 2014a) were

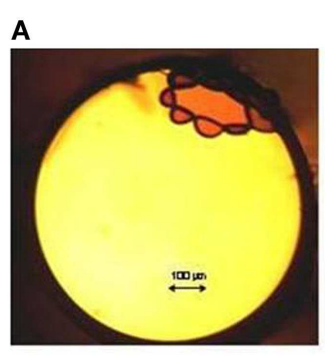

D

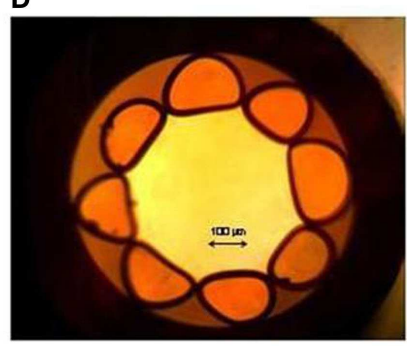

B

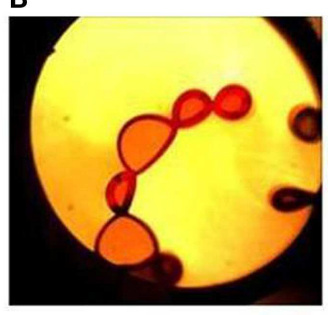

C

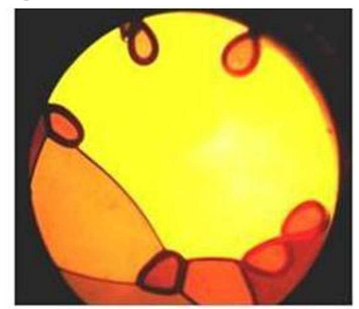

E

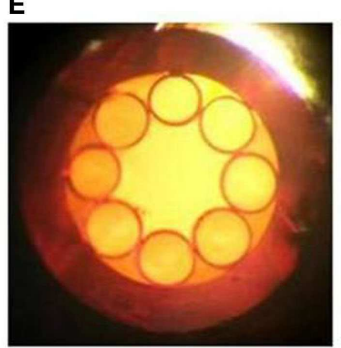

FIGURE 10 |The cross-sections of $\mathrm{As}_{2} \mathrm{~S}_{3}$ MOFs obtained under different drawing conditions. The temperature: (A-C) $330-340^{\circ} \mathrm{C}$, (D) $320^{\circ} \mathrm{C}$, (E) $310^{\circ} \mathrm{C}$. The excess argon pressure in capillaries: (A) 0, (B) $100 \mathrm{~Pa}$, (C) $600 \mathrm{~Pa}$, (D) $1000 \mathrm{~Pa}$, (E) $4000 \mathrm{~Pa}$ (Shiryaev et al., 2014b). 
measured by the cut-back method on a Bruker IFS-113v Fourier transform IR spectrometer. The measured length of optical fibers was from 1 to $2 \mathrm{~m}$. In this experiment, a radiation propagated not only through the air-core but also through the substrate tube and the cladding capillaries. To remove the influence of cladding modes, a liquid gallium-indium immersing alloy was applied on the surface of input and output ends of fiber.

The spectra of optical losses of prepared $\mathrm{As}_{2} \mathrm{~S}_{3}$ NCHCFs with diameter of $700 \mu \mathrm{m}$ illustrated in Figures 10D,E are given in Figure 11 (Shiryaev et al., 2014b). The optical losses in fibers without Ga-In immersion were higher than in Ga-In immersed fibers. Our best fiber was transparent in the $1.5-8.2 \mu \mathrm{m}$ wavelength range and has minimum loss about $3 \mathrm{~dB} / \mathrm{m}$ at $4.8 \mu \mathrm{m}$. Unfortunately, the obtained MOFs contained the absorption bands of impurity associated with arsenic sulfide. There are some bands due to $\mathrm{S}-\mathrm{H}$ bonds at $6.8 ; 4.1 ; 3.7 ; 3.1 \mu \mathrm{m}$; due to $\mathrm{CO}_{2}$ impurity at 4.31 and $4.34 \mu \mathrm{m}$; due to $\mathrm{OH}$ groups at $2.92 \mu \mathrm{m}$; due to $\mathrm{COS}$ at $4.9 \mu \mathrm{m}$; and due to molecular $\mathrm{H}_{2} \mathrm{O}$ at $6.33 \mu \mathrm{m}$.

The spectrum of optical losses of prepared $\mathrm{As}_{2} \mathrm{~S}_{3} \mathrm{NCHCF}$ with 10 capillaries is given in Figure 12. The minimum loss in this fiber was about $1.2 \mathrm{~dB} / \mathrm{m}$ in the range of $2.7-3.4 \mu \mathrm{m}$.

Investigation of the as-prepared hollow-core microstructured fibers based on $\mathrm{As}_{30} \mathrm{Se}_{50} \mathrm{Te}_{20}$ and $\mathrm{As}_{40} \mathrm{~S}_{30} \mathrm{Se}_{30}$ glasses has shown their transparency in the range of $2-11 \mu \mathrm{m}$ and possibility to transfer of the $\mathrm{CO}_{2}$-laser radiation. Minimum optical loss in As-Se-Te glass hollow-core PCF was about $4 \mathrm{~dB} / \mathrm{m}$ at $9.5 \mu \mathrm{m}(6.5 \mathrm{~dB} / \mathrm{m}$ at $10.6 \mu \mathrm{m}$ ) (Figure 13) (Kosolapov et al., 2011), and one in As-S-Se glass hollow-core PCF was $30 \mathrm{~dB} / \mathrm{m}$ at $10.6 \mu \mathrm{m}$ and $10 \mathrm{~dB} / \mathrm{m}$ at $2 \mu \mathrm{m}$ ) (Shiryaev and Churbanov, 2013).

To investigate the distribution of the $\mathrm{CO}_{2}$-laser radiation intensity over the fiber cross-section, a $\mathrm{ZnSe}$ lens was used to launch the radiation into the fiber, while the intensity distribution over the fiber output endface was observed with the help of an

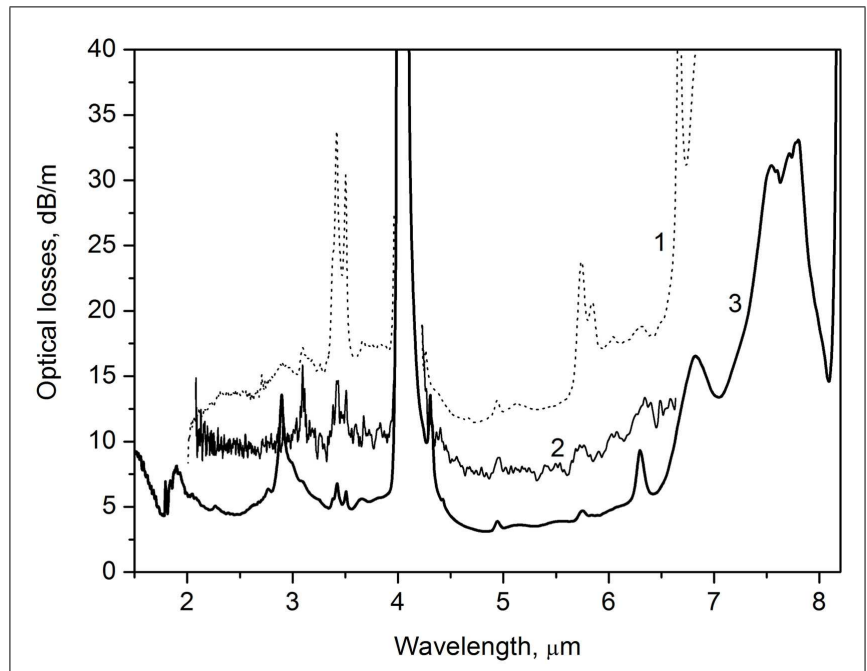

FIGURE 11 |The spectra of optical losses in prepared $\mathrm{As}_{2} \mathrm{~S}_{3}$ NCHCFs with diameter of $700 \mu \mathrm{m}$ : (1) fiber illustrated in Figure 10D without Ga immersion; (2) fiber illustrated in Figure 10D with Ga-In immersion; (3) fiber illustrated in Figure 10E with Ga-In immersion (Shiryaev et al., 2014b).
Electrophysics PV320 thermal imaging camera. It was found that a much greater power can be transmitted through the core, which means that the light propagating through the glass owing to the total internal reflection experiences much stronger attenuation. The distribution in Figure $\mathbf{1 4}$ obtained under proper excitation conditions shows that the $\mathrm{CO}_{2}$-laser radiation is well confined in the hollow-core (Kosolapov et al., 2011).

\section{AGING OF CHALCOGENIDE PCFs}

The efficiency and the stability of chalcogenide glass microstructured optical fibers are limited by the shift of their optical properties that occurs over time due to an aging process. The optical aging on $\mathrm{As}_{2} \mathrm{~S}_{3}$ microstructured optical fiber upon exposure to air was reported in papers (Toupin et al., 2014; Mouawad et al., 2014).

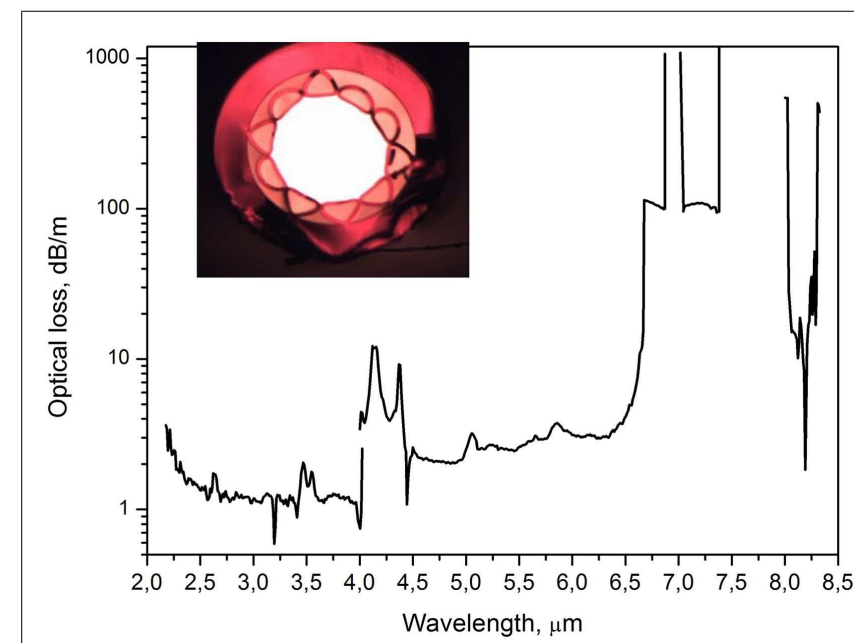

FIGURE 12 | Spectrum of optical losses of as-prepared $\mathrm{As}_{2} \mathrm{~S}_{3} \mathrm{NCHCF}$ with 10 capillaries

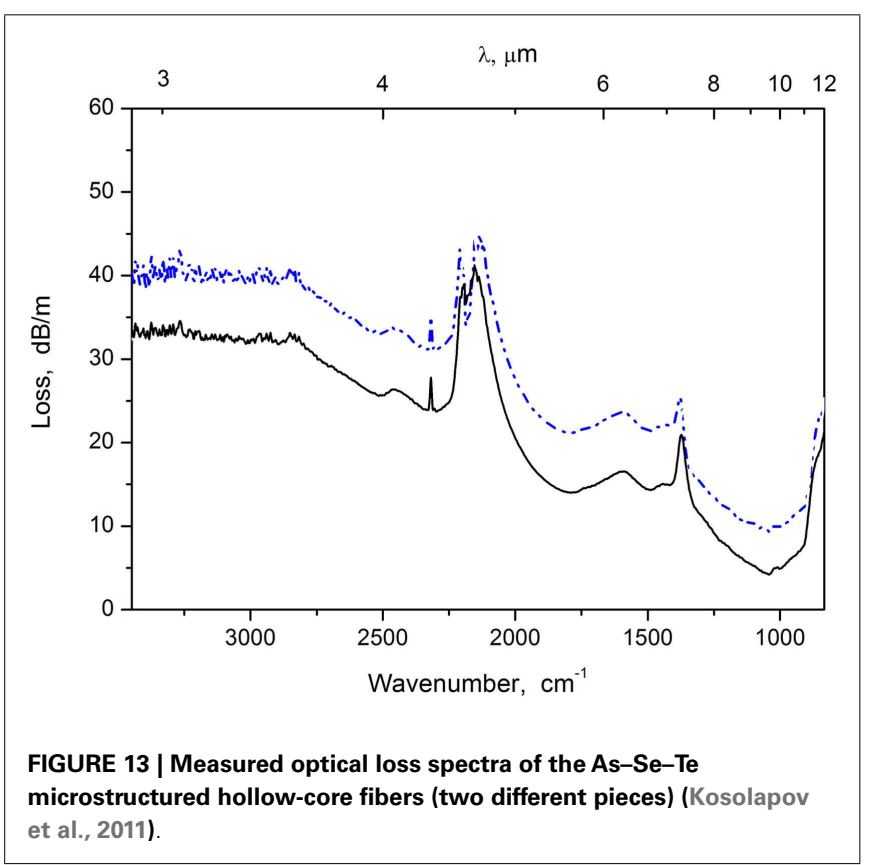



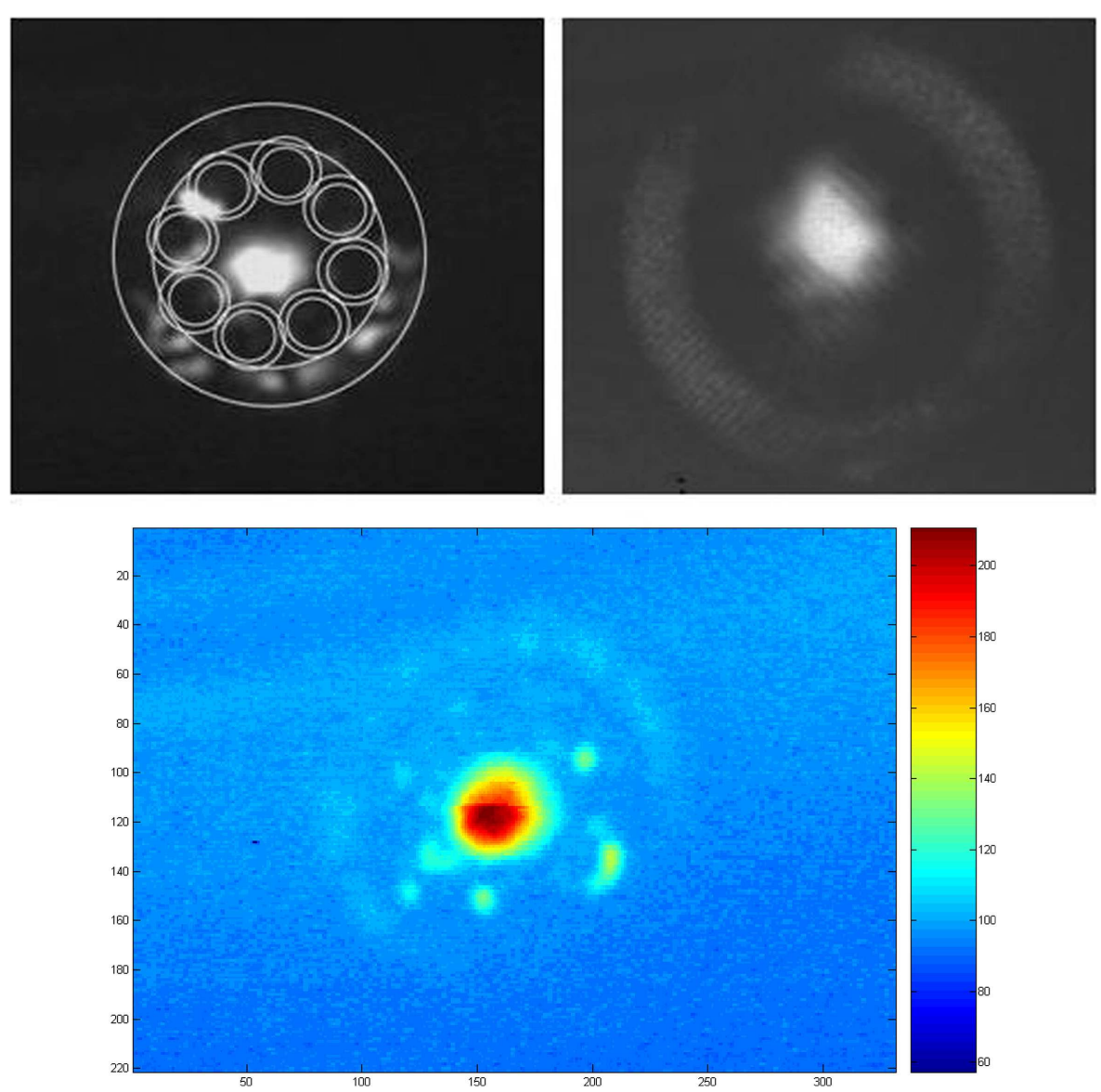

FIGURE 14 | Intensity distribution of $\mathrm{CO}_{2}$-laser radiation over the fiber cross-section (Kosolapov et al., 2011).

The optical aging was established to associate with a dynamic grow of $\mathrm{OH}$ and $\mathrm{H}_{2} \mathrm{O}$ attributed absorption bands.

Paper (Toupin et al., 2014) describes the optical aging of MOFs prepared from four glass compositions ( $\mathrm{As}_{30} \mathrm{Se}_{50} \mathrm{Te}_{20}, \mathrm{As}_{38} \mathrm{Se}_{62}$, $\mathrm{Ge}_{10} \mathrm{As}_{22} \mathrm{Se}_{68}$, and $\left.\mathrm{As}_{40} \mathrm{~S}_{60}\right)$ and stored in air. The results have shown that the same absorption bands associated with the presence of $\mathrm{OH}$ hydroxyl groups and molecular water appear whatever the glass composition of the fiber. The continuous evolution of the concentrations of hydroxyl groups in the MOFs as a function of exposure period was observed. These changes upon exposure period have shown that the harmful effect of atmospheric moisture in the holes of the MOF occurs rapidly over the first few hours and is the most important in the early centimeters of the MOF. The content of hydroxyl groups decreases exponentially with distance away from the MOF extremity. However, the growing rate of absorption peaks depends on the glass composition. Optical aging as a function of fiber composition was summarized as: $\mathrm{As}_{40} \mathrm{~S}_{60}<\mathrm{Ge}_{10} \mathrm{As}_{22} \mathrm{Se}_{68}<\mathrm{As}_{38} \mathrm{Se}_{62}<\mathrm{As}_{30} \mathrm{Se}_{50} \mathrm{Te}_{20}$. The value of "optical contamination pollution rate" at $6.3 \mu \mathrm{m}$ wavelength was estimated to be $0.16,0.02,0.001$, and $0.0003 \mathrm{~dB} / \mathrm{m} /$ day for $\mathrm{As}_{40} \mathrm{~S}_{60}$, $\mathrm{Ge}_{10} \mathrm{As}_{22} \mathrm{Se}_{68}, \mathrm{As}_{38} \mathrm{Se}_{62}$, and $\mathrm{As}_{30} \mathrm{Se}_{50} \mathrm{Te}_{20}$, respectively.

The authors of paper (Mouawad et al., 2014) have demonstrated the deleterious time evolution of $\mathrm{As}_{2} \mathrm{~S}_{3} \mathrm{MOF}$ upon exposure of the MOFs core to atmospheric moisture. FTIR experiments have shown that an increase in the $\mathrm{OH}$ and $\mathrm{SH}$ content in the MOFs causes extra losses. The exposure of $\mathrm{As}_{2} \mathrm{~S}_{3}$ glass to atmospheric conditions was determined to induce the formation and the growth of pyramidal defects on the glass surface. These surface structures are $\mathrm{As}_{2} \mathrm{O}_{3}$ crystals according to the XDS results and arise from reaction with the atmosphere. Substantial increase in the intensity of $\mathrm{OH}$ absorption bands occurring over the first hours requires the specific storage of $\mathrm{As}_{2} \mathrm{~S}_{3} \mathrm{MOF}$ in dry conditions, immediately after the drawing process. To decrease the chemical aging process of the MOF core, a protection of the MOF holes from diffusion of atmospheric steam is required. For that purpose, the authors (Mouawad et al., 2014) have airproofed the fiber ends by means of a methacrylate-based polymer. The fiber ends were soaked in the liquid polymeric solution, and then let to polymerize in free atmosphere at room temperature.

\section{SUMMARY}

The review on the recent developments on chalcogenide glass HC-MOFs demonstrates their attractive perspectives for mid-IR applications, as well as the trends and challenges of the technique of manufacturing. 
The calculation of the structural design of chalcogenide NCHCF has shown the possibility of achieving optical losses less than $1 \mathrm{~dB} / \mathrm{km}$ in the mid-IR spectral region. NCHCFs with a hollow-core are promising especially for the transmission of high optical power.

Preparation of chalcogenide glass capillaries by extrusion from a double crucible, in contrast to commonly used capillary drawing of a tubular preform, eliminates the additional step of heat treatment of the sample to reduce the crystallization and to obtain the smooth outer and inner surfaces of the capillaries.

The prepared NCHCFs of chalcogenide glass were suitable for the mid-IR radiation transmission, although the optical losses were higher than the theoretically predicted level.

The achievement of optical losses close to the theoretically calculated level is determined by not only the high purity of glass on impurities and its optical homogeneity, but also the high accuracy of the geometrical dimensions of a given photonic crystal structure.

Thus, to prepare the hollow-core microstructured fibers with low optical losses in the mid-IR range (special interest in fibers transmitted at wavelengths of 5-6 and 9.3-10.6 $\mu \mathrm{m}$ ), further optimization of their design and fiber drawing conditions is required to prevent the deformation of their photonic crystal structure, which is also one of the reasons for the increase in optical losses.

\section{REFERENCES}

Benabid, F. (2006). Hollow-core photonic bandgap fibre: new light guidance for new science and technology. Phil. Trans. R. Soc. A 364, 3439-3462. doi:10.1098/rsta. 2006.1908

Benabid, F., Couny, F., Knight, J. C., Birks, T. A., and Russell, P. (2005). Compact, stable and efficient all-fibre gas cells using hollow-core photonic crystal fibres. Nature 434, 488-491. doi:10.1038/nature03349

Birks, T. A., Knight, J. C., and Russell, P. (1997). Endlessly single-mode photonic crystal fiber. Opt. Lett. 22, 961-963. doi:10.1364/OL.22.000961

Brilland, L., Smektala, F., Renversez, G., Chartier, T., Troles, J., Nguyen, T., et al. (2006). Fabrication of complex structures of holey fibers in chalcogenide glass. Opt. Express 14, 1280-1285. doi:10.1364/OE.14.001280

Conseil, C., Coulombier, Q., Boussard-Pledel, C., Troles, J., Brilland, L., Renversez, G., et al. (2011). Chalcogenide step index and microstructured single mode fibers. J. Non Cryst. Solids 357, 2480-2483. doi:10.1016/j.jnoncrysol.2010.11.090

Coulombier, Q., Brilland, L., Houizot, P., Chartier, T., N'Guyen, T. N., Smektala, F., et al. (2010). Casting method for producing low-loss chalcogenide microstructured optical fibers. Opt. Express 18, 9107-9112. doi:10.1364/OE.18. 009107

Cregan, R. F., Mangan, B. J., Knight, J. C., Birks, T. A., Russell, P. J., Roberts, P. J., et al. (1999). Single-mode photonic band gap guidance of light in air. Science 285, 1537-1539. doi:10.1126/science.285.5433.1537

Desevedavy, F., Renversez, G., Brilland, L., Houizot, P., Troles, J., Coulombier, Q., et al. (2008). Small-core chalcogenide microstructured fibers for the infrared. Appl. Opt. 47, 6014-6021. doi:10.1364/AO.47.006014

Desevedavy, F., Renversez, G., Troles, J., Houizot, P., Brilland, L., Vasilief, I., et al. (2010). Chalcogenide glass hollow core photonic crystal fibers. Opt. Mater. 32, 1532-1539. doi:10.1016/j.optmat.2010.06.016

El-Amraoui, M., Fatome, J., Jules, J. C., Kibler, B., Gadret, G., Fortier, C., et al. (2010). Strong infrared spectral broadening in low-loss As-S chalcogenide suspended core microstructured optical fibers. Opt. Express 18, 4547-4556. doi:10.1364/OE.18.004547

Gibson, D. J., and Harrington, J. A. (2004). Extrusion of hollow waveguide performs with a one-dimensional photonic bandgap structure. J. Appl. Phys. 95, 3895-3900. doi:10.1063/1.1667277

He, J., Xiong, C., Clark, A., Collins, M., Gai, X., Choi, D., et al. (2012). Effect of lowRaman window position on correlated photon-pair generation in a chalcogenide $\mathrm{Ge}_{11.5} \mathrm{As}_{24} \mathrm{Se}_{64.5}$ nanowire. J. Appl. Phys. 112, 1-5. doi:10.1063/1.4769740
Hu, K., Kabakova, I., Buttner, T., Lefrancois, S., Hudson, D., He, S., et al. (2014). Low-threshold Brillouin laser at 2 mum based on suspended-core chalcogenide fiber. Opt. Lett. 39, 4651-4654. doi:10.1364/OL.39.004651

Jiang, X., Euser, T. G., Abdolvand, A., Babic, F., Tani, F., Joly, N. Y., et al. (2011). Singlemode hollow-core photonic crystal fiber made from soft glass. Opt. Express 19, 15438-15444. doi:10.1364/OE.19.015438

Katsuyama, T., and Matsumura, H. (1986). Low loss Te-based chalcogenide glass optical fibers. Appl. Phys. Lett. 49, 22-23. doi:10.1063/1.97089

Knight, J. C., Birks, T. A., Russell, P. J., and Atkin, D. M. (1996). All-silica single-mode fiber with photonic crystal cladding. Opt. Lett. 21, 1547-1549. doi:10.1364/OL.21.001547

Knight, J. C., Birks, T. A., Russell, P. J., and Sandro, J. P. (1998). Properties of photonic crystal fiber and the effective index model. J. Opt. Soc. Am. A 15, 748-752. doi:10.1364/JOSAA.15.000748

Kosolapov, A. F., Pryamikov, A. D., Biriukov, A. S., Shiryaev, V. S., Astapovich, M. S., Philippovsky, D. V., et al. (2011). Demonstration of $\mathrm{CO}_{2}$ laser power delivery through chalcogenide glass fiber with hollow negative-curvature core. Opt. Express 19, 25723-25728. doi:10.1364/OE.19.025723

Monro, T. M., Richardson, D. J., Broderick, N. G. R., and Bennett, P. J. (1999). Holey optical fibers: an efficient modal model. J. Lightwave Technol. 17, 1093-1101. doi:10.1109/50.769313

Monro, T. M., West, Y. D., Hewak, D. W., Broderick, N. G. R., and Richardson, D. J. (2000). Chalcogenide holey fibres. Electron. Lett. 36, 1998-2000. doi:10.1049/el:20001394

Mouawad, O., Strutynski, C., Picot-Clémente, J., and Désévédavy, F. (2014). Optical aging behaviour naturally induced on $\mathrm{As}_{2} \mathrm{~S}_{3}$ microstructured optical fibres. Opt. Mater. Express 4, 2190-2203. doi:10.1364/OME.4.002190

Nishii, J., Morimoto, S., Inagawa, I., Iizuka, R., Yamashita, T., and Yamagishi, T. (1992). Recent advances and trends in chalcogenide glass fiber technology: a review. J. Non Cryst. Solids 140, 199-208. doi:10.1016/S0022-3093(05)80767-7

Pryamikov, A. D., Biriukov, A. S., Kosolapov, A. F., Plotnichenko, V. G., Semjonov, S. L., and Dianov, E. M. (2011). Demonstration of a waveguide regime for a silica hollow - core microstructured optical fiber with a negative curvature of the core boundary in the spectral region $>3.5 \mu \mathrm{m}$. Opt. Express 19, 1441-1448. doi:10.1364/OE.19.001441

Pryamikov, A. D., Kosolapov, A. F., Plotnichenko, V. G., and Dianov, E. M. (2012). "Transmission of CO2 laser radiation through glass hollow core microstructured fibers," in $\mathrm{CO}_{2}$ Laser - Optimisation and Application, ed. D. C. Dumitras (InTech). Available from: http://www.intechopen. com/books/co2-laser-optimisation-and-application/transmission-of-co2-laserradiationthrough-glass-hollow-core-microstructured-fibers

Revathi, S., Inbathini, S. R., and Saifudeen, R. A. (2014). Highly nonlinear and birefringent spiral photonic crystal fiber. Adv. Optoelectron. 2014, 6. doi:10.1155/ 2014/464391

Shiryaev, V. S., Adam, J.-L., Zhang, X. H., Boussard-Plédel, C., Lucas, J., and Churbanov, M. F. (2004). Infrared fibers based on Te-As-Se glass system with low optical losses. J. Non Cryst. Solids 336, 113-119. doi:10.1016/j.jnoncrysol.2004.01.006

Shiryaev, V. S., and Balda, R. (2006). "Preparation of high purity chalcogenide glasses and fibers," in Photonic Glasses, ed. R. Balda (Kerala, India: Research Signpost), 151-195.

Shiryaev, V. S., and Churbanov, M. F. (2013). Trends and prospects of development of chalcogenide fibers for mid infrared transmission. J. Non Cryst. Solids 377, 225-230. doi:10.1016/j.jnoncrysol.2012.12.048

Shiryaev, V. S., Churbanov, M. F., Dianov, E. M., Plotnichenko, V. G., Adam, J.-L., and Lucas, J. (2005). Recent progress in preparation of chalcogenide As-Se-Te glasses with low impurity content. J. Optoelectron. Adv. Mater. 7, 1773-1779.

Shiryaev, V. S., Kosolapov, A. F., Pryamikov, A. D., Snopatin, G. E., Churbanov, M. F., Biriukov, A. S., et al. (2014a). Development of technique for preparation of $\mathrm{As}_{2} \mathrm{~S}_{3}$ glass preforms for hollow core microstructured optical fibers. J. Optoelectron. Adv. Mater. 16, 1020-1025.

Shiryaev, V. S., Mishinov, S. V., and Churbanov, M. F. (2014b). Adhesion of glassy arsenic sulfide to quartz glass. Inorg. Mater. 50, 1157-1161. doi:10.1134/ S0020168514110181

Shiryaev, V. S., Mishinov, S. V., and Churbanov, M. F. (2015). Investigation of adhesion of chalcogenide glasses to silica glass. J. Non Cryst. Solids. 408, 71-75. doi:10.1016/j.jnoncrysol.2014.10.010

Skryabin, D. V., and Wadsworth, W. J. (2010). "Nonlinear optics and solitons in photonic crystal fibres," in Nonlinearities in Periodic Structures and Metamaterials, 
Springer Series in Optical Sciences, Vol. 150, eds C. Denz, S. Flach, and Y. S. Kivshar (Berlin: Springer), 37-54.

Snopatin, G. E., Churbanov, M. F., Pushkin, A. A., Gerasimenko, V. V., Dianov, E. M., and Plotnichenko, V. G. (2009a). High purity arsenic-sulfide glasses and fibers with minimum attenuation of $12 \mathrm{~dB} / \mathrm{km}$. J. Opt. Adv. Mater. Rapid Commun. 3, 669-671.

Snopatin, G. E., Shiryaev, V. S., Plotnichenko, G. E., Dianov, E. M., and Churbanov, M. F. (2009b). High-Purity Chalcogenide Glasses for Fiber Optics. Inorg. Mater. 45, 1439-1460. doi:10.1134/S0020168509130019

Temelkuran, B., Hart, S. D., Benoit, G., Joannopoulos, J. D., and Fink, Y. (2002). Wavelength-scalable hollow optical fibres with large photonic bandgaps for CO2 laser transmission. Nature 420, 650-653. doi:10.1038/ nature 01275

Toupin, P., Brilland, L., Mechin, D., Adam, J.-L., and Troles, J. (2014). Optical aging of chalcogenide microstructured optical fibers. J. Lightwave Technol. 32, 2428-2432. doi:10.1109/JLT.2014.2326461

Wadsworth, W. J., Knight, J. C., Ortigosa-Blanch, A., Arriaga, J., Silvestre, E., and Russell, P. S. J. (2001). Soliton effects in photonic crystal fibres at $850 \mathrm{~nm}$. Electron. Lett. 36, 53-55. doi:10.1049/el:20000134

Wei, C., Kuis, R., Chenard, F., and Hu, J. (2014). "Chalcogenide negative curvature hollow-core photonic crystal fibers with low loss and low power ratio in the glass," in CLEO: 2014, Laser Science to Photonic Applications Technical Conference (San Jose, CA: SM1N), 5.
Yablonovitch, E. (1987). Inhibited spontaneous emission in solid-state physics and electronics. Phys. Rev. Lett. 58, 2059-2062. doi:10.1103/PhysRevLett.58.2059

Yeh, P., Yariv, A., and Marom, E. (1978). Theory of Bragg fiber. J. Opt. Soc. Am. 68, 1196-1201. doi:10.1364/JOSA.68.001196

Zhang, Y., Schroeder, J., Husko, C., Lefrancois, S., Choi, D., Madden, S., et al. (2014). Pump degenerate phase-sensitive amplification in chalcogenide waveguides. J. Opt. Soc. Am. B Opt. Phys. 31, 780-787. doi:10.1364/JOSAB.31.000780

Conflict of Interest Statement: The author declares that the research was conducted in the absence of any commercial or financial relationships that could be construed as a potential conflict of interest.

Received: 14 January 2015; accepted: 07 March 2015; published online: 24 March 2015. Citation: Shiryaev VS (2015) Chalcogenide glass hollow-core microstructured optical fibers. Front. Mater. 2:24. doi: 10.3389/fmats.2015.00024

This article was submitted to Optics and Photonics, a section of the journal Frontiers in Materials.

Copyright () 2015 Shiryaev. This is an open-access article distributed under the terms of the Creative Commons Attribution License (CC BY). The use, distribution or reproduction in other forums is permitted, provided the original author(s) or licensor are credited and that the original publication in this journal is cited, in accordance with accepted academic practice. No use, distribution or reproduction is permitted which does not comply with these terms. 